\title{
RNASET2 impairs the sperm motility via PKA/PI3K/calcium signal pathways
}

\author{
Yali Xu ${ }^{1, *}$, Yong Fan ${ }^{1,2, *}$, Weimin Fan ${ }^{1,3, *}$, Jia Jing ${ }^{1}$, Ke Xue ${ }^{1}$, Xing Zhang ${ }^{4}$, Bin Ye ${ }^{4}$, Yingjie $\mathrm{Ji}^{4}$, \\ Yue Liu ${ }^{1}$ and Zhide Ding ${ }^{1}$ \\ ${ }^{1}$ Department of Histology, Embryology, Genetics and Developmental Biology, Shanghai Key Laboratory for \\ Reproductive Medicine, School of Medicine, Shanghai Jiao Tong University, Shanghai, China, ${ }^{2}$ Department of \\ Assisted Reproduction, Shanghai Ninth People's Hospital, School of Medicine, Shanghai Jiao Tong University, \\ Shanghai, China, ${ }^{3}$ Reproductive Medicine Center, Shanghai Ruijin Hospital, School of Medicine, Shanghai Jiao Tong \\ University, Shanghai, China and ${ }^{4}$ Department of Clinical Medicine, School of Medicine, Shanghai Jiao Tong \\ University, Shanghai, China
}

Correspondence should be addressed to Y Liu or Z Ding; Email: liuyue@shsmu.edu.cn or zding@shsmu.edu.cn

$*(\mathrm{Y} X \mathrm{Xu}, \mathrm{Y}$ Fan and $\mathrm{W}$ Fan contributed equally to this work $)$

\begin{abstract}
Asthenozoospermia is one of the leading causes of male infertility owing to a decline in sperm motility. Herein, we determined if there is a correlation between RNASET2 content on human spermatozoa and sperm motility in 205 semen samples from both asthenozoospermia patients and normozoospermia individuals. RNASET2 content was higher in sperm from asthenozoospermia patients than in normozoospermia individuals. On the other hand, its content was inversely correlated with sperm motility as well as progressive motility. Moreover, the inhibitory effect of RNASET2 on sperm motility was induced by incubating normozoospermic sperm with RNase T2 protein. Such treatment caused significant declines in intracellular spermatozoa PKA activity, PI3K activity and calcium level, which resulted in severely impaired sperm motility, and the sperm motility was largely rescued by cAMP supplementation. Finally, protein immunoprecipitation and mass spectrometry identified proteins whose interactions with RNASET2 were associated with declines in human spermatozoa motility. AKAP4, a protein regulating PKA activity, coimmunoprecipated with RNASET2 and they colocalized with one another in the sperm tail, which might contribute to reduced sperm motility. Thus, RNASET2 may be a novel biomarker of asthenozoospermia. Increases in RNASET2 can interact with AKAP4 in human sperm tail and subsequently reduce sperm motility by suppressing PKA/PI3K/calcium signaling pathways.

Reproduction (2018) 155 383-392
\end{abstract}

\section{Introduction}

Ribonucleases (RNases) are crucial enzymes controlling RNA metabolism in almost all organisms. Traditionally, RNases are subdivided into the RNase T1, RNase $\mathrm{A}$ and RNase T2 families, according to their base sequence specificity, structure, function, optimal $\mathrm{pH}$ and origin (Campomenosi et al. 2006, Luhtala \& Parker 2010). The RNases in the T2 family mainly include ACTIBIND in fungi and RNASET2 in humans. Indeed, ACTIBIND and RNASET2 share $34 \%$ and $52 \%$ amino acid sequence homology and have similar biological activities in RNA metabolism and particularly in tumor suppression. Recent studies reported that ACTIBIND, a T2 ribonuclease from Aspergillus niger, can bind to actin and disrupt its internal networks in colon cancer cells and induce cell motility inhibition as well as have antiangiogenic and anticarcinogenic effects (Roiz et al. 2006, Schwartz et al. 2007, de Leeuw et al. 2012).
Similarly, RNASET2, the only member of the RNases T2 family in humans, has also been implicated to possess antitumorigenic and antiangiogenic activities (Acquati et al. 2005, 2011, Smirnoff et al. 2006, Monti et al. 2008). Deletion of the chromosomal region in the RNASET2 gene is associated with several human malignancies (Lin \& Morin 2001, Monti et al. 2008, Acquati et al. 2013). More importantly, serial successive studies reported that both the natural RNase T2 protein isolated from Aspergillus niger and the recombinant RNase T2 protein expressed either in E. coli system or eukaryotic cells disrupted the organization of intracellular actin filaments and manifest antitumorigenic and antiangiogenic activities in both in vitro and in vivo assays (Nesiel-Nuttman et al. 2014, 2015, Roiz et al. 2016).

Besides demonstrating its ability to inhibit invasiveness of cancer cells, our previous study showed that RNASET2 is also present in human semen and is 
presumed to be an asthenozoospermia diagnostic marker (Liu et al. 2013). We found that RNASET2 was localized in the acrosome, neck and tail regions of human spermatozoa, and moreover, it was highly expressed in asthenozoospermic spermatozoa and highly localized in the neck and tail region, which provides sperm motility through flagellum undulation. The current study was undertaken to further characterize the potential correlation between RNASET2 content and asthenozoospermia and in particular to focus on characterizing the RNASET2-linked signaling pathways inhibiting human sperm motility.

Herein, we report on the human spermatozoa RNASET2 content in 205 individual semen samples obtained from 100 normozoospermia men and 105 asthenozoospermia patients. Initially, we confirmed that RNASET2 content is higher in the spermatozoa from asthenozoospermia patients. Besides its binding to actin, RNase T2 also combines with AKAP4 in the sperm tail and reduces sperm motility through declines in the PKA/PI3K/calcium signal pathway activity.

\section{Materials and methods \\ Specimen procurement}

Human semen specimens were obtained from the Reproductive Medicine Center, Ruijin Hospital, Shanghai Jiao Tong University, School of Medicine. Enrollment occurred between June, 2013 and December, 2014. Use of the semen samples was approved by the Ethics Committee of these units, and all experiments were performed in accordance with relevant guidelines and regulations. All semen specimens, both normozoospermia and asthenozoospermia, were collected from participants (20-35 years old) who gave written informed consent, and then were analyzed using computer-assisted semen analyzer (CASA, Hamilton-Thorn Research, Beverly, MA, USA). The semen specimens were selected according to World Health Organization guidelines (WHO 2010), i.e. sperm density was higher than $1.5 \times 10^{7} \mathrm{sperm} / \mathrm{mL}$ and normal spermatozoa morphology was higher than $4 \%$.

\section{Flow cytometry assay}

Detection of RNASET2 on spermatozoa was carried out by indirect immunofluorescent staining in conjunction with flow cytometry. The immunofluorescent staining and flow cytometry assay were performed as described (Liu et al. 2013). Spermatozoa were separated by centrifugation $\left(500 \mathrm{~g}, 4^{\circ} \mathrm{C}\right.$, $15 \mathrm{~min}$ ) and fixed in $4 \%$ paraformaldehyde for $10 \mathrm{~min}$ at room temperature. Sperm samples were labeled with mouse antiRNase T2 monoclonal antibody (1:200 dilution, sc393729, Santa Cruz Biotechnology) and subsequently with CF488conjugated donkey anti-mouse $\operatorname{lgG}$ (1:400 dilution, Biotium, Hayward, CA, USA). Flow cytometry measured the spermatozoa fluorescence signals (Becton Dickinson, Beckman Coulter, Brea, CA, USA). Cell Quest software analyzed the emission originating from at least 30,000 events (Beckman Coulter).

\section{Sperm motility inhibition assays}

In a sperm motility inhibition assay, RNase T2 protein (secreted by Aspergillus niger, also named ACTIBIND) was purified from the Aspergillus niger growth medium as described (Roiz et al. 2000, Liu et al. 2013). The purified RNase T2 protein was finally dissolved in PBS and its identity was previously confirmed by Western blot and mass spectrometry in our previous study (Liu et al. 2013). The BCA assay determined purified RNase T2 concentration (Pierce).

The sperm motility inhibition assay was carried out as previously reported (Liu et al. 2013). Spermatozoa were obtained by the swim-up method in Tyrode's buffer (SigmaAldrich). The isolated sperm suspension density was adjusted to 30 million sperm $/ \mathrm{mL}$ and then divided into three groups: (1) RNase T2 group incubated with $100 \mu \mathrm{g} / \mathrm{mL}$ of RNase T2 protein, (2) control group incubated with $100 \mu \mathrm{g} / \mathrm{mL}$ of RNase A and (3) blank group in Tyrode's buffer without any treatment. Sperm suspensions were cultured at $5 \% \mathrm{CO}_{2}, 37^{\circ} \mathrm{C}$ for 0.5 , 1 and $2 \mathrm{~h}$. CASA determined inhibitory effectiveness after treatment with $100 \mu \mathrm{g} / \mathrm{mL}$ of RNase T2 protein in accordance with 2010 WHO criteria for making this assessment.

\section{Protein immunoprecipitation (IP) assay}

Immunoprecipitation was applied to separate the proteins interacting with RNASET2 in human spermatozoa. Human spermatozoa were lysed with RIPA buffer (Pierce) supplemented with protease inhibitor (Roche, Werk Penzberg) and kept in a rotating ice bath for at least $30 \mathrm{~min}$ in order to allow proteins to dissolve completely. Centrifugation $(12,000 \mathrm{~g}, 10 \mathrm{~min})$ was then applied to remove the sediment, and $500 \mu \mathrm{L}$ of a sperm protein sample were incubated with $10 \mu \mathrm{g}$ of rabbit antiRNase T2 antibodies (ab107313, Abcam) overnight at $4{ }^{\circ} \mathrm{C}$. Additionally, normal rabbit IgG (Millipore) was used as a negative control. Then, $50 \mu \mathrm{L}$ of Protein $\mathrm{G}$ agarose were added into the slurry containing the sperm-antibody complex and incubated with gentle mixing for $4 \mathrm{~h}$ at $4^{\circ} \mathrm{C}$. After incubation, the agarose beads were washed with TBS by centrifugation three times and the agarose beads with protein complex were further analyzed by either liquid chromatography tandem mass spectrometry (LC-MS) or Western blot.

\section{LC-MS analysis}

The LC-MS analysis was performed as described (Liu et al. 2015). Reverse phase-high performance liquid chromatography (RP-HPLC) separation was achieved on the Suveyer HPLC system (Thermo Fisher Scientific) equipped with a self-packed column $(75 \mu \mathrm{m} \times 120 \mathrm{~mm})$ at a flow rate of $250 \mathrm{~nL} / \mathrm{min}$. An LTQOrbitrap instrument (Thermo Fisher Scientific) was operated in data-dependent mode. The mass spectrometer was set so that each full MS scan was followed by 10 most intense ions for MS/MS with charge $\geq+2$ and the following Dynamic Exclusion settings were used: repeat counts, 1; repeat duration, 120s; exclusion duration, $180 \mathrm{~s}$. The full mass was scanned in the Orbitrap analyzer with $R=60,000$ (defined at $\mathrm{m} / \mathrm{z} 400$ ), and the subsequent MS/MS analysis was performed in the LTQ analyzer. All MS/MS spectra were searched using Maxquant 
software against the human International Protein Index (IPI) database (version 3.68).

\section{Western blot analysis}

Protein samples were separated by SDS-PAGE, and then transferred to polyvinylidene difluoride (PVDF) membranes (Millipore) using a semi-dry transfer apparatus (Bio-Rad); membranes were blocked with 5\% BSA in TBS containing $0.2 \%$ Tween-20. Immunoblotting was performed with either mouse anti-RNase T2 monoclonal antibody (Santa Cruz, sc393729) or anti-AKAP4 monoclonal antibody (Abcam, ab56551) at a 1:2000 dilution. Normal mouse IgG (Millipore) was used as a negative control at the same dilution, followed by incubation with goat anti-mouse IgG-conjugated to horseradish peroxidase (HRP) (Jackson ImmunoResearch) at a 1:10,000 dilution. Signals were detected by enhanced chemiluminescence (Millipore) according to the manufacturers' protocol.

\section{Indirect immunofluorescence assay}

Indirect immunofluorescent staining identified RNASET2 and AKAP4 co-localization in human spermatozoa. Sperm was separated from semen samples by a discontinuous $47 / 90 \%$ Percoll density gradient centrifugation at $800 \mathrm{~g}$ for $30 \mathrm{~min}$ at $4^{\circ} \mathrm{C}$. Subsequently, sperm smears were prepared and fixed with pre-cooled acetone for $15 \mathrm{~min}$. All subsequent incubations were performed in a humidified chamber. Then, the sperm smears were blocked with 5\% BSA in PBS for 30 min at room temperature, and later incubated with a mixture of purified rabbit anti-RNASET2 polyclonal antibodies (Abcam, ab107313, 1:100 diluted in PBS) and mouse anti-AKAP4 monoclonal antibodies (Abcam, ab56551, 1:200 dilution) overnight at $4^{\circ} \mathrm{C}$. Meanwhile, the mixture consisted of normal mouse IgG (Millipore; 1:50 dilution) and normal rabbit IgG (Millipore; 1:100 dilution) was used as negative control. After washing, the sperm samples were incubated with a mixture of CF555-labeled donkey anti-mouse IgG (Biotium, 1:400 dilution) and CF488-labeled donkey anti-rabbit IgG (Biotium, $1: 400$ dilution) for $1 \mathrm{~h}$ at $37^{\circ} \mathrm{C}$. Finally, the fluorescent-stained sperm samples were detected under a laser scanning confocal microscope (LSCM, Carl Zeiss LSM-510) by excitation at $488 \mathrm{~nm}$ and $543 \mathrm{~nm}$ and monitoring emission at 500-550 nm and $565-615 \mathrm{~nm}$. The immunofluorescence test was repeated three times and semen from four normozoospermia donors was combined for each co-localization experiment.

\section{PKA and PI3K assays}

In order to detect the levels of PKA and PI3K (phosphoinositide 3-kinase) in human spermatozoa, three individual samples from donors were combined to guarantee that there were almost $3 \times 10^{6}$ spermatozoa in every experiment and each could be repeated three times. A Swim-up spermatozoa mixture was divided into three parts and incubated with RNase T2, RNase A and Tyrode's solution, respectively for $1.5 \mathrm{~h}$ at $37^{\circ} \mathrm{C}$. Then, the samples were washed twice in cold PBS and resuspended in $200 \mu \mathrm{L}$ cell lysis buffer followed by ultrasonic decomposition of the sperm. Finally, sperm lysates were centrifuged at $800 \mathrm{~g}$ for $10 \mathrm{~min}$ at $4^{\circ} \mathrm{C}$, and the supernatant was collected for further analysis.

Intracellular PKA and PI3K were detected by using assay kits according to the manufacturer's protocol. PKA level was detected by employing a ProFluor PKA assay kit from Promega. At first, a standard kinase reaction started with a provided bisamide rhodamine 110 peptide substrate (PKA R110 substrate) in the provided reaction buffer. In this configuration, the PKA R110 substrate is non-fluorescent. The kinase reaction was performed following its termination with a termination buffer containing a protease reagent. Amino acids were removed from the non-phosphorylated PKA R110 substrate, causing the production of highly fluorescent rhodamine 110, which was measured in a luminescence spectrometer (Perkin Elmer LS50). Nevertheless, the phosphorylated PKA R110 substrate was resistant to digestion by the protease reagent and remained non-fluorescent. Therefore, the fluorescence intensity detected in the assay is inversely correlated with kinase activity. PI3K activity was determined by using a kit from Promega. The lipid kinase reaction was performed by incubating lipid substrate PIP2:3PS with a recombinant enzyme and ATP, and the kinase activity was measured using the ADP-Glo kinase assay. Firstly, after the kinase reaction, an ATP depletion reagent was added to terminate the reaction and deplete any remaining ATP, leaving only ADP. Then, a detection reagent was added to simultaneously convert ADP to ATP allowing the newly synthesized ATP to be converted to light by using a coupled luciferase reaction measured via a FB 12 luminometer (Berthold, Germany).

\section{Intracellular $\mathrm{Ca}^{2+}$ measurements}

Swim-up spermatozoa suspensions from donors (there are nearly $3 \times 10^{6}$ spermatozoa) were treated with RNase T2, RNase A and Tyrode's solution respectively for $1.5 \mathrm{~h}$ at $37^{\circ} \mathrm{C}$. Then, every sample was incubated with Fluo-4, AM (1:1000) (Dojindo, Kumamoto, Japan) for $30 \mathrm{~min}$. Calcium fluorescence was detected using a luminescence spectrometer (Perkin Elmer LS50), and read at an excitation wavelength of $506 \mathrm{~nm}$ and an emission wavelength of $526 \mathrm{~nm}$.

\section{Sperm motility rescue assays}

Sperm suspensions of donors were incubated with $100 \mu \mathrm{g} / \mathrm{mL}$ RNase T2 protein for $1 \mathrm{~h}$ and then supplemented with $10 \mu \mathrm{M}$ db-cAMP (dibutyryl cAMP, Sigma-Aldrich) for another $0.5 \mathrm{~h}$. CASA determined rescue effectiveness $0.5 \mathrm{~h}$ after $10 \mu \mathrm{M}$ cAMP supplementation in accordance with 2010 WHO criteria for making this assessment.

\section{Statistical analysis}

Statistical analysis was carried out using SAS 8.2 software. The data referring to sperm motility are reported as mean \pm S.E.M. and the other data as mean \pm S.D. Group comparisons were made using Student's t-test where appropriate. One-way analysis of variance (ANOVA) test was used assuming a two-tail hypothesis with $P<0.05$. The correlations of mean fluorescent intensity and sperm motility or progressive motility 
were assessed by Spearman correlation analysis. $P$ values of $<0.05$ were considered statistically significant.

\section{Results}

Negative correlations among RNASET2 expression and spermatozoa motility and progressive motility

Sperm parameters, especially for the sperm motility, sperm count and normal sperm morphology in the semen from both normozoospermia donors and asthenozoospermia patients used in this experiment were calculated in accordance with 2010 WHO criteria (Data not shown).

To assess the RNASET2 content in human sperm, the mean fluorescence of RNASET2 positive sperm was scored using flow cytometry and correlated with sperm motility and progressive motility using linear regression analysis. As shown in Fig. 1A, the expression of RNASET2, indicated by the mean fluorescence of positive cells, was significantly higher in the sperm from asthenozoospermia patients $(n=105,36.75 \pm 1.33)$ than that in the sperm from normal donors $(n=100$, $29.58 \pm 0.76, P<0.01$, Fig. $1 \mathrm{~A})$.

The correlation analysis showed that the mean fluorescence was negatively correlated with sperm motility ( $n=205, r=-0.264, P<0.001$, Fig. 1B) and progressive motility, respectively $(n=205, r=-0.242$, $P<0.001$, Fig. 1C).

Compared with our previous study (Liu et al. 2013), the current study contains enlarged sample size (205 sperm samples) to further prove that RNASET2 content is higher in sperm from asthenozoospermia patients.

\section{RNase T2 treatment reduces sperm motility and progressive motility}

To determine the effect of RNase T2 on sperm motility, motile human sperm from donors was incubated with either $100 \mu \mathrm{g} / \mathrm{mL}$ purified RNase T2 protein, or $100 \mu \mathrm{g} /$ $\mathrm{mL}$ RNase A (control) or Tyrode's buffer (blank) for $0.5,1,2 \mathrm{~h}$, respectively, and then sperm motility and progressive motility were analyzed by CASA methods. Compared with the sperm treated with either Tyrode's buffer (0.5 h: $83.39 \pm 1.51, \quad 1 \mathrm{~h}: 79.23 \pm 1.97,2 \mathrm{~h}$ : $74.37 \pm 2.23)$ or RNase A $(0.5 \mathrm{~h}: 77.98 \pm 1.67,1 \mathrm{~h}$ : $72.36 \pm 1.83,2 \mathrm{~h}: 68.38 \pm 2.33)$, RNase T2 treatment (0.5 h: $59.56 \pm 2.94,1$ h: $51.42 \pm 4.21,2$ h: $32.87 \pm 3.90)$ significantly decreased sperm motility $(n=52, P<0.01$, Fig. 2A). Similarly, RNase T2 treatment also significantly reduced sperm progressive motility at $0.5 \mathrm{~h}$ (RNase T2 treatment: $16.54 \pm 1.53$, blank: $32.14 \pm 1.95$, RNase A treatment: $28.46 \pm 1.72$ ), $1 \mathrm{~h}$ (RNase T2 treatment, $14.94 \pm 1.81$, blank, $31.92 \pm 1.61$, RNase A treatment: $27.88 \pm 1.61)$ and $2 \mathrm{~h}$ (RNase T2 treatment, 9.79 \pm 1.50 , blank, 30.62 \pm 1.78 , RNase A treatment: $27.33 \pm 1.58$ ) $(n=52, P<0.01$, Fig. 2B). Apparently, these data show that RNase T2 significantly impairs both sperm motility and progressive motility.

Likewise, compared with our previous study (Liu et al. 2013), the current study also contains enlarged sample size (52 sperm samples in the protein inhibitory test) to further prove that RNase T2 treatment reduces sperm motility and progressive motility.

\section{Identification of proteins interacting with RNASET2 in human spermatozoa}

To elucidate the underlying mechanism whereby RNASET2 reduces both sperm motility and progressive motility, human spermatozoa proteins interacting with RNASET2 were isolated by colP and identified by MS analysis. MS identified several interacting proteins isolated by IP with an anti-RNASET2 monoclonal antibody (Table 1). RNASET2, coprecipitated with actin, a well-known binding target of RNASET2 in several cell lines. Moreover, this antibody efficiently pulled down the A-kinase anchor protein 4 (AKAP4) along with RNASET2. Notably, AKAP4 has been reported to affect sperm motility by modifying PKA activity (Miki et al. 2002, Eddy et al. 2003, Luconi et al. 2011), which is consistent with our finding that RNase T2 could combine with AKAP4 thereby blocking the effect of AKAP4, and thereby suppressing sperm PKA activity.

Figure 3A provides the results of Western blot analysis showing that RNASET2 interacts with AKAP4. This is evident because RNASET2 and
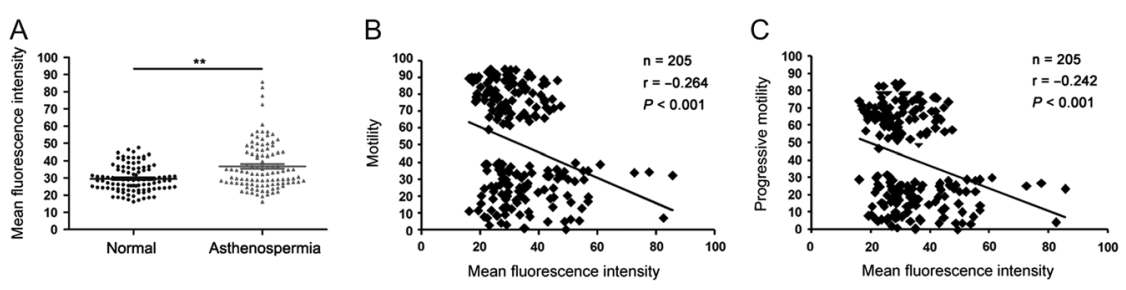

Figure 1 RNase T2 expression and its correlation with sperm motility and progressive motility in normal individuals and asthenozoospermia patients. (A) Flow cytometric fluorescence intensity assessment of RNase T2 expression showed that RNase T2 levels are higher in asthenozoospermia patients $(n=105)$ than those in normal individuals $(n=100)$. Correlations between mean fluorescence intensity and sperm motility (B) and progressive motility (C) were calculated by Spearman correlation analysis. Data are expressed as mean \pm S.E.M. ${ }^{* *} P<0.01$. 

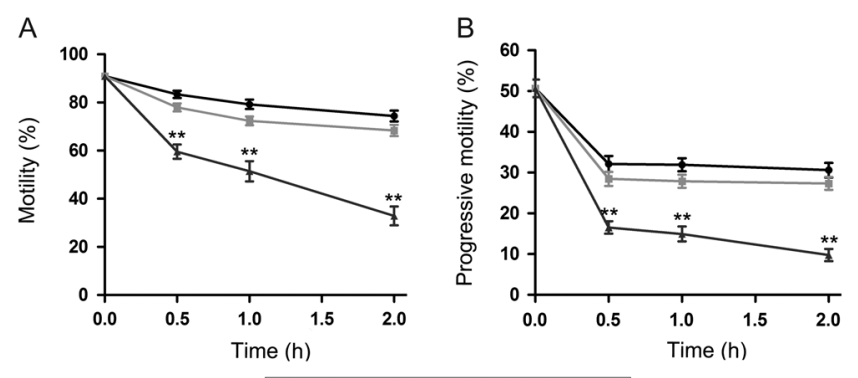

$\rightarrow$ Blank $\rightarrow$ Control $\leftarrow$ RNase T2

Figure 2 Purified RNase T2 treatment reduces sperm motility and progressive motility. After being incubated with either $100 \mu \mathrm{g} / \mathrm{mL}$ purified RNase T2, or RNase A (control) or Tyrode's solution (blank) respectively, for $0.5,1$ and $2 \mathrm{~h}$, sperm motility (A) and progressive motility (B) were determined by CASA. Data are expressed as mean \pm S.E.M., and calculated by ANOVA test. ${ }^{* *} P<0.01$.

AKAP4 co-immunoprecipitate with either the antiRNASET2 or anti-AKAP4 antibody in pull downs of co-immunoprecipitates of human spermatozoa extracts. Furthermore, immunofluorescence staining also identified RNASET2 co-localization with AKAP4 on human spermatozoa, especially in the principle piece of the tail region (Fig. 3B). These data make it very likely that RNASET2 interacts with AKAP4 in human spermatozoa.

\section{Sperm signaling pathways mediating RNase T2-induced motility declines}

Our results provide substantive indications that RNASET2 interacts with AKAP4 in human sperm. It is an abundant structural protein anchoring the PKA regulatory subunit to the fibrous sheath and thereby regulating PKA activity. To confirm that such an interaction accounts for how RNASET2 and AKAP4 affect PKA activity, we determined if PKA kinase and PI3K signaling pathways mediate RNase T2 suppression of human sperm motility and progressive motility. As shown in Fig. 4A $(n=10$, $P<0.01$ ), the RNASET2 content was inversely correlated with PKA activity. Its activity was significantly lower in sperm treated with RNase T2 because the fluorescence intensity is inversely correlated with kinase activity $\left(285.30 \pm 38.06\right.$ per $10^{6}$ sperm), whereas in sperm treated with either RNase A, it fell to $256.04 \pm 59.53$ per $10^{6}$ sperm or $244.92 \pm 42.41$ per $10^{6}$ sperm in Tyrode's solution. These results show that RNase T2 treatment clearly reduced PKA activity. With regards to PI3K activity incubated with RNase T2, its activity was $1.13 \times 10^{6} \pm 0.31 \times 10^{6}$ per $10^{6}$ sperm. This level was significantly lower than that in sperm treated with either RNase A $\left(2.78 \times 10^{6} \pm 1.62 \times 10^{6}\right.$ per $10^{6}$ sperm $)$ or Tyrode's solution only $\left(3.07 \times 10^{6} \pm 1.75 \times 10^{6}\right.$ per $10^{6}$ sperm) (Fig. 4B, $n=10, P<0.01$ ).

Similarly, intracellular calcium levels and PKA and PI3K activity declined together in spermatozoa treated with RNase T2 (318.91 \pm 39.35 per $10^{6}$ sperm) compared with those in spermatozoa treated with either RNase A $\left(354.58 \pm 45.42\right.$ per $10^{6}$ sperm $)$ or Tyrode's solution $\left(364.14 \pm 49.14\right.$ per $10^{6}$ sperm) (Fig. 4 C, $\left.n=10, P<0.01\right)$.

\section{CAMP supplementation rescues $R$ Nase T2-induced declines in sperm motility}

To evaluate whether or not the PKA-PI3K signaling pathway contributes to mediating the inhibitory effect of RNase T2 content on sperm motility, we determined if cAMP supplementation, a known PKA activator, rescued RNase T2-induced declines in sperm motility. RNase T2 exposure for $1 \mathrm{~h}$ decreased the sperm motility from $91.75 \pm 1.31$ to $49.45 \pm 5.45(n=20, P<0.01)$, whereas supplementation with $10 \mu \mathrm{M}$ db-cAMP for $30 \mathrm{~min}$ increased it to $78.45 \pm 3.33(n=20, P<0.01$, Fig. 5A). Likewise, RNase $\mathrm{T} 2$ exposure caused progressive motility to decline from $42.65 \pm 4.55$ to $19.80 \pm 2.73(n=20$, $P<0.01)$, whereas $10 \mu \mathrm{M}$ db-cAMP supplementation partially reversed this decline by increasing it to $32.20 \pm 2.33$ ( $n=20, P<0.01$, Fig. 5B).

\section{Discussion}

In a previous study, we identified RNASET2 expression in human semen and suggested that it has a potential role in modulating sperm motility in asthenozoospermia (Liu et al. 2013). In the current study, we confirmed that RNASET2 content on human spermatozoa negatively correlates with declines in sperm motility because

Table 1 Human spermatozoa proteins interacting with RNASET2 identified by IP and MS.

\begin{tabular}{|c|c|c|c|c|c|c|c|}
\hline Protein names & Gene names & Peptides & $\begin{array}{l}\text { Unique } \\
\text { peptides }\end{array}$ & $\begin{array}{c}\text { Sequence } \\
\text { coverage }(\%)\end{array}$ & Mol. weight (kDa) & Score & $\begin{array}{c}\text { MS/MS } \\
\text { count }\end{array}$ \\
\hline Fibronectin & FN1 & 18 & 18 & 9.7 & 243.23 & 161.99 & 21 \\
\hline Ribonuclease T2 & RNASET2 & 11 & 11 & 37.4 & 29.215 & 132.3 & 22 \\
\hline A-kinase anchor protein 4 & AKAP4 & 15 & 15 & 21.8 & 93.444 & 112.34 & 16 \\
\hline Heat shock-related $70 \mathrm{kDa}$ protein 2 & HSPA2 & 13 & 11 & 25.7 & 70.02 & 111.22 & 18 \\
\hline Polyadenylate-binding protein 1 & PABPC1 & 9 & 9 & 15.7 & 65.747 & 103.42 & 13 \\
\hline Clusterin & CLU & 9 & 9 & 28.4 & 48.803 & 84.52 & 12 \\
\hline Heat shock protein HSP 90-alpha & HSP90AA1 & 10 & 6 & 16.1 & 84.659 & 84.305 & 11 \\
\hline Actin, cytoplasmic 2 & ACTG1 & 9 & 2 & 24.5 & 41.792 & 78.814 & 13 \\
\hline Protein S100-A9 & S100A9 & 5 & 5 & 51.8 & 13.242 & 72.939 & 9 \\
\hline Endoplasmin & HSP90B1 & 8 & 8 & 10.8 & 92.468 & 66.662 & 10 \\
\hline
\end{tabular}



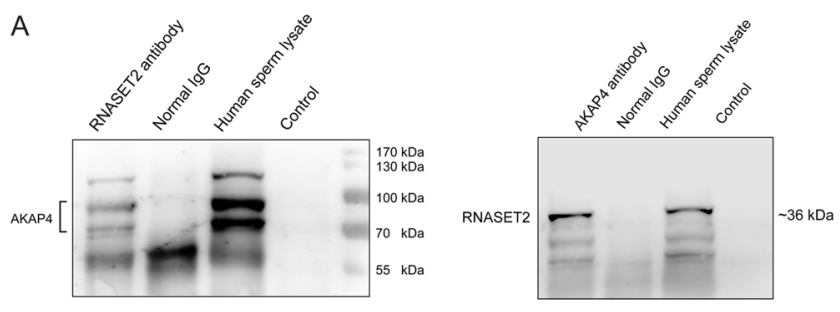

B
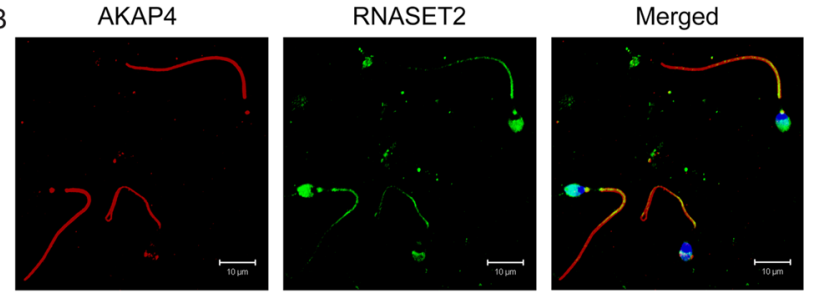

Figure 3 AKAP4 interaction with RNASET2 (A) Western blots of AKAP4 and RNASET2 in immunoprecipitates of human spermatozoa extracts, indicate that they co-immunoprecipitate with one another. (B) Immunofluorescent staining of AKAP4 and RNASET2 in human spermatozoa showed that they colocalize on the principle piece of the tail region.

we gained access to a larger semen sample size. One hundred normozoospermia semen samples and 105 asthenozoospermia semen samples were collected and analyzed by flow cytometry assay enabling us to measure the fluorescence of at least of 30,000 spermatozoa in each semen sample and determine their RNASET2 content. The results show that RNASET2 content increased in sperm collected from asthenozoospermia patients, which was associated with corresponding declines in both sperm motility and progressive motility in each semen sample. Accordingly, our results show that there is an inverse relationship between RNASET2 content and both sperm motility and progressive motility in 205 sperm samples. This association suggests that increased RNASET2 content in sperm from asthenozoospermia patients might be an important factor accounting for declines in sperm motility in these patients.

This finding prompted us to confirm its validity by using the RNase T2 protein treatment test to determine if RNase T2 altered sperm motility. ACTIBIND was used for this purpose, which is analogous to RNase T2. This substitution was made because ACTIBIND can be isolated in large amounts in its active form from Aspergillus niger. It was isolated from conditioned Aspergillus niger culture medium by a two-step anionexchange chromatographic method even though the full-length human recombinant RNASET2 may be more appropriate for this type of experiment. Nevertheless, this alternative was chosen because RNASET2 yields were quite low from a yeast expression system or mammalian cell expression system possibly due to ribonuclease degradative activity. This concern is perhaps mitigated by the fact that RNase T2 family members are ubiquitous and evolutionarily conserved. Thus, the amino acid sequence or structure as well as biological activity of ACTIBIND and RNASET2 have high homology (Luhtala \& Parker 2010). In our previous study, the RNaseT2 protein identity purified from Aspergillus niger was confirmed by Western blot analysis and MS prior to its use (Liu et al. 2013). We found that either 200, 500 or $1000 \mu \mathrm{g} / \mathrm{mL}$ RNase T2 protein efficiently reduced both sperm motility and progressive motility. However, there were only 23 samples in this previous study whereas 52 samples were used in the sperm motility assay in the current study. This larger sample size was sufficient for us to confirm that sperm motility and especially progressive motility rapidly decreased within $30 \mathrm{~min}$ after being treated with RNase T2 protein $(100 \mu \mathrm{g} / \mathrm{mL})$. It also could probably underlie why RNASET2 had a significant inhibitory effect on sperm motility. Meanwhile, NesielNuttman and colleagues reported that a truncated form of recombinant RNASET2 expressed in E. coli, with a short non-glycolyslated protein fragment possesses
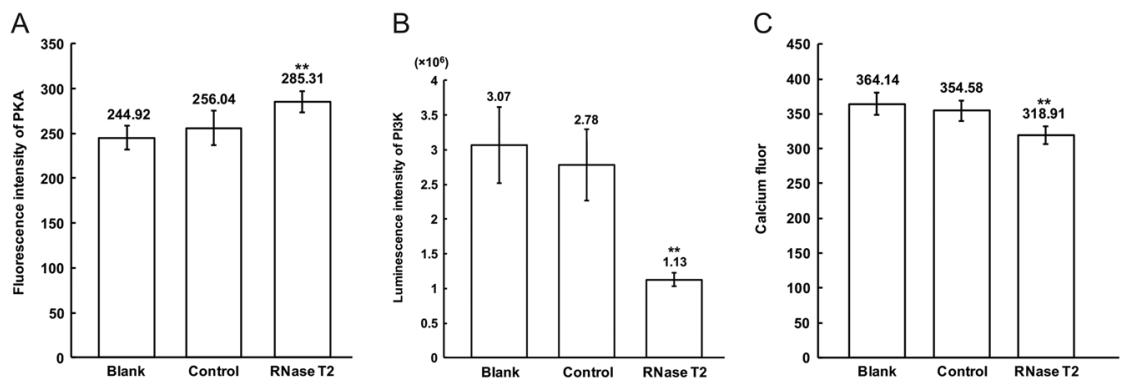

Figure 4 Intracellular $\mathrm{PKA}, \mathrm{PI} 3 \mathrm{~K}$ and $\mathrm{Ca}^{2+}$ level measurements. (A) PKA activity assays were performed following treatments of human spermatozoa with either RNase T2, or RNase A (Control) and or Tyrode's solution (Blank), respectively. The fluorescence intensity measured in the assay is inversely correlated with kinase activity, showed reduced PKA activity in RNase T2 treatment. (B) PI3K activity assay. The fluorescence intensity is proportional to the relative concentration of activated $\mathrm{PI} 3 \mathrm{~K}$, showed reduced $\mathrm{PI} 3 \mathrm{~K}$ activity in RNase T2 treatment. (C) Intracellular $\mathrm{Ca}^{2+}$ concentration was measured using a $\mathrm{Ca}^{2+}$ signaling probe. Relative $\mathrm{Ca}^{2+}$ levels in swim-up sperm incubated with RNase T2 decreased in comparison with those in sperm treated instead with either RNase A or Tyrode's solution. ${ }^{*} P<0.05,{ }^{* *} P<0.01$ respectively. 


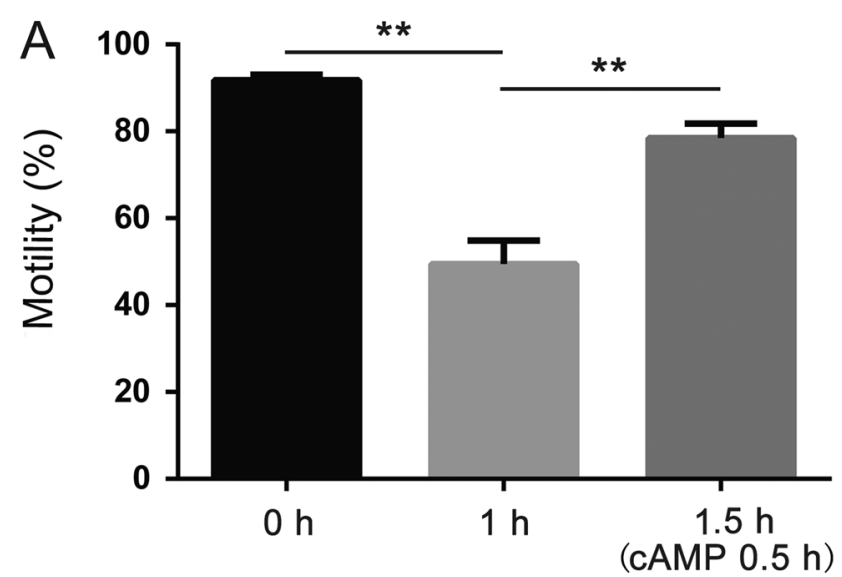

RNase T2 treatment

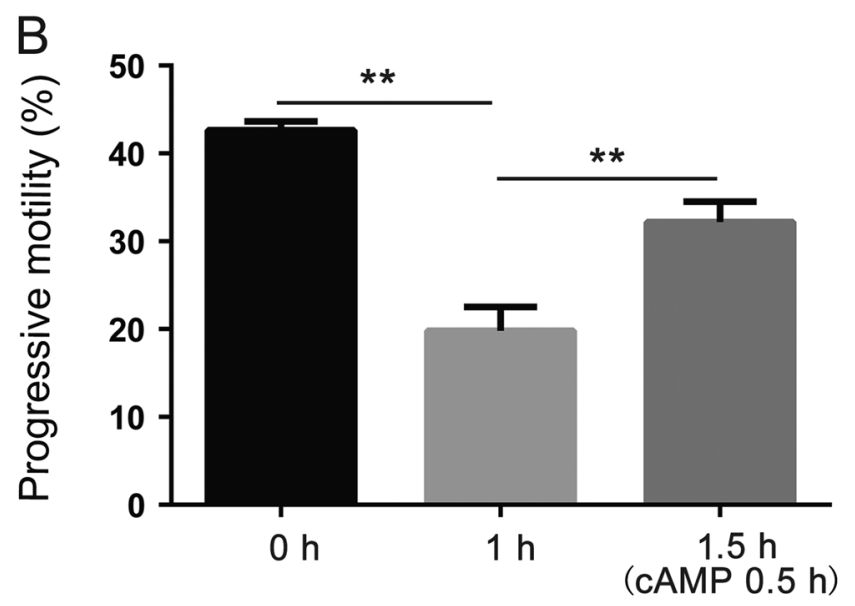

RNase T2 treatment

Figure 5 db-cAMP rescues RNase T2-induced suppression of sperm motility. The declines in sperm motility (A) and progressive motility (B) were largely rescued by $10 \mu \mathrm{M} \mathrm{db}$-cAMP supplementation for another $0.5 \mathrm{~h}$. Data are expressed as mean \pm S.E.M., and ANOVA evaluated significance. ${ }^{* *} P<0.01$.

actin-binding capacity and displays antitumorigenic and antiangiogenic activities in both in vitro and in vivo assays (Nesiel-Nuttman et al. 2014). Although a large quantity of activated RNase T2 protein could be conveniently purified from Aspergillus niger, a truncated recombinant protein fragment was used because we predicted that it might be a more efficient and accurate approach for our future undertakings determining the contribution by RNASET2 content to controlling sperm motility and its development as a molecular drug target for use in male contraception.

It is generally accepted that good sperm motility is crucial to normal male fertility and semen quality, whereas poor sperm motility or immotile spermatozoa reduce fertility. Most mammalian sperm display two types of motility: (1) activated motility in ejaculated sperm and (2) hyperactivated motility in sperm recovered from the site of fertilization (Overstreet et al. 1980, Suarez \&
Osman 1987). Sperm motility maintenance is dependent on some complex interactions between different cell signaling systems; one of them is the signal pathway network involved in mediating sperm movement. The cAMP/PKA and calcium signaling pathway complex are usually recognized to mediate such control. On the one hand, the PKA-dependent phosphorylation of flagella proteins is responsible for activating sperm motility in mammals (Tash \& Means 1982, 1983), i.e. the activation of PKA induced by cAMP phosphorylates some kinases such as phosphatidylinositol 3-kinase (PI3K) and Src family protein tyrosine kinase (SFK), and flagellum proteins, such as calcium-binding tyrosine phosphorylation-regulated protein (CABYR) and then initiates flagella motility (Turner 2006). Meanwhile, the activation of PKA also increases in turn the activity of calcium channels such as CatSper leading to increases in intracellular $\mathrm{Ca}^{2+}$ level. Thus, PKA-dependent phosphorylation undisputedly plays a regulatory role in sperm motility. On the other hand, calcium is necessary for acquiring motility in mammalian spermatozoa. In general, there are at least two different calcium signaling pathways. One of them is calmodulin (CaM) independent and changes in intracellular calcium levels modulate soluble adenylyl cyclase (sAC) activity. Increases in its activity in turn generate cAMP leading to PKA stimulation, which then is directly linked to promoting flagella function (Buck et al. 1999, Chen et al. 2000, Jaiswal \& Conti 2003, Litvin et al. 2003, Esposito et al. 2004, Hess et al. 2005). The other $\mathrm{Ca}^{2+}$ signaling pathway is instead CaM dependent and increases in intracellular $\mathrm{Ca}^{2+}$ activate calmodulin kinase (CaMK), which then induces spermatozoa motility (Wu et al. 2000, Ignotz \& Suarez 2005, Marin-Briggiler et al. 2005). Besides, the lipid signaling phosphatidylinositol 3-kinase (PI3K) pathway is also suggested to be a key regulator of sperm motility or capacitation. PI3K can activate AKT via PIP3 (phosphatidylinositol 3,4,5-triphosphate), i.e., PI3K initially activates PIP3 and then PIP3 combines with PDK1 (phosphoinositide-dependent kinase 1) and AKT, and subsequently, PIP3 prompts PDK1 to catalyze AKT phosphorylation. This reaction maintains sperm motility by inhibiting caspases 3 and caspases 7 (Gallardo Bolanos et al. 2014).

In the current study, we definitively found that incubation of motile sperm with RNase T2 efficiently reduced intracellular PKA activity, PI3K activity and declines in intracellular calcium influx. Coincidently, the inhibitory effect of RNase T2 on sperm motility could be largely rescued by db-cAMP supplementation, a cellpermeable cAMP analogue that activates PKA. Thus, the increases in RNASET2 content appear to decrease human sperm motility by reducing intracellular PKA PI3K activities and lowering intracellular calcium levels. It appears likely that one of the factors contributing to lower sperm motility and progressive motility in asthenozoospermia is elevated spermatozoa RNASET2 


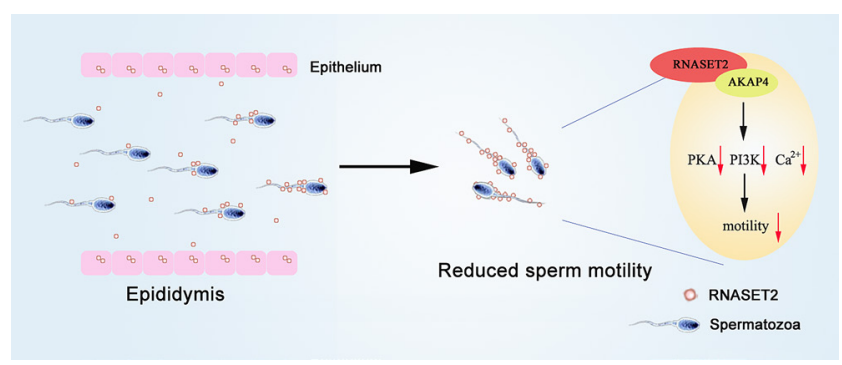

Figure 6Schematic model describing RNASET2 impairment of human sperm motility.

content because it reduces PKA-mediated protein phosphorylation and lowers intracellular calcium levels, both of which contribute to the control of sperm motility.

Nevertheless, the underlying mechanism accounting for how changes in RNASET2 content contribute to controlling sperm motility and progressive motility are not fully elucidated. It is generally known that RNASET2 can directly bind to cell surface actin and disrupt intracellular actin networks in cancerous cell lines. In our previous study, we also confirmed that RNASET2 binds to actin in human spermatozoa (Liu et al. 2013). In addition, we presumed that the effects of RNASET2 on sperm motility were likely involved in its ability to target some signal pathway regulator rather than merely bind to actin. Thus, in this study, the proteins interacting with RNASET2 were isolated by protein IP and identified by LC-MS in human spermatozoa. MS results showed actually that some of the proteins interacting with RNASET2 belong to the actin family. Furthermore, it was of great interest to us that large amounts of AKAP4 precipitated with RNASET2 suggesting that it has a potential role in signal pathway regulation. Such interaction was further confirmed by the finding that RNASET2 and AKAP4 co-immunoprecipitate with either the anti-RNASET2 or anti-AKAP4 antibody. In addition to IP and immunoblot, indirect immunofluorescence analyses demonstrated AKAP4 and RNASET2 localization in human spermatozoa. Staining of AKAP4 was mainly localized in the sperm neck and tail, especially in the principle piece of sperm flagellum, while RNASET2 accumulated/stuck in the acrosome region of the sperm head, neck and the principle piece of sperm tail. Moreover, significant RNASET2 and AKAP4 co-localization was found in the principle piece of the sperm tail. Notably, human spermatozoa are highly polarized cells, and their motility is fully dependent on sperm tail activity. Thus, their specific co-localization and interaction in the sperm tail strongly indicate their involvement in contributing to sperm motility regulation.

As reported, AKAP4 is an abundant structural protein present in the sperm fibrous sheath, which enables it to anchor regulatory subunits of PKA to the fibrous sheath. This is requisite for PKA activation to increase sperm protein tyrosine phosphorylation status, which in turn leads to activation of the tail beat (Eddy et al. 2003, Luconi et al. 2011). Akap4-null male mice are infertile, due to the sperm having a defective fibrous sheath and subsequent progressive loss in motility (Miki et al. 2002). Interestingly, the function of AKAP4 in regulating PKA activity and sperm motility is consistent with our results showing that RNase T2 treatment can reduce sperm motility through declines in PKA/PI3K/calcium signaling pathway activation. These findings suggest that increases in RNASET2 content downregulate human sperm motility by binding and interacting with AKAP4 in the principle piece of the spermatozoa, which then directly reduces PKA activity. Such declines lower the phosphorylation status of various target proteins that are presumed to initiate several signaling pathways including increases in PI3K activity and intracellular calcium transients.

This study provides new insights into the function of RNASET2 that might be related to regulating AKAP4 activity. However, the interaction between the two proteins is not only limited to controlling sperm motility. In relationship to cancer, RNASET2 levels are relevant to several different types of malignancy. Loss of RNASET2 has been associated with a variety of solid neoplasms, such as ovarian tumors, carcinomas of the breast, melanoma, colon/rectum tumor and hematologic malignancies (Lin \& Morin 2001, Acquati et al. 2005, 2011, 2013, Smirnoff et al. 2006, Monti et al. 2008). Meanwhile, AKAP4 is expressed not only in human germ cells, but is also used as a new cancer/testis antigen in various tumor cells. AKAP4 overexpression correlates with differences in tumor malignancy, such as glioblastoma, myeloma, prostate cancers, breast cancer, cervical cancer, ovarian cancer, colon cancer and esophageal cancer (Ragel et al. 2007, Chiriva-Internati et al. 2008, 2012, Agarwal et al. 2013, Saini et al. 2013, Gumireddy et al. 2015, Jagadish et al. 2016, Li et al. 2016). This similar association between declines in RNASET2 content and increases in AKAP4 content in tumor genesis, combined with their documented interaction described in this study, strengthens the notion that both these proteins may contribute to tumor genesis and progression and sperm motility as well.

In conclusion, we found that the RNASET2 content in spermatozoa was higher in asthenozoospermia than that in normozoospermic samples. Besides its documented direct binding to cell surface actin and disruption of actin networks in spermatozoa, excessive RNase T2 content in human sperm may largely reduce the levels of intracellular PKA, PI3K, protein phosphorylation and intracellular calcium levels through binding and interacting with AKAP4. Such a decline could reduce intracellular PKA- and PI3K-mediated increases in protein phosphorylation status. Furthermore, rises in intracellular calcium levels are crucial for sperm motility activation, whereas declines in the aforementioned levels eventually results in poor sperm motility (Fig. 6). 
Therefore, our results support the notion that RNASET2 is a critical factor whose modulation affects human sperm motility and also has the potential to be a relevant biomarker for evaluating sperm quality in a clinical setting.

\section{Declaration of interest}

The authors declare that there is no conflict of interest that could be perceived as prejudicing the impartiality of the research reported.

\section{Funding}

This research project was supported by grants from National Natural Science Foundation of China (No. 81370752 and No. 81571487), the Science and Technology Commission of Shanghai Municipality (No. 16ZR1418600).

\section{Acknowledgements}

The authors thank Prof. Peter Reinach for his editorial assistance.

\section{References}

Acquati F, Possati L, Ferrante L, Campomenosi P, Talevi S, Bardelli S, Margiotta C, Russo A, Bortoletto E, Rocchetti R et al. 2005 Tumor and metastasis suppression by the human RNASET2 gene. International Journal of Oncology 26 1159-1168.

Acquati F, Bertilaccio S, Grimaldi A, Monti L, Cinquetti R, Bonetti P, Lualdi M, Vidalino L, Fabbri M, Sacco MG et al. 2011 Microenvironmental control of malignancy exerted by RNASET2, a widely conserved extracellular RNase. PNAS 108 1104-1109. (https://doi.org/10.1073/ pnas.1013746108)

Acquati F, Lualdi M, Bertilaccio S, Monti L, Turconi G, Fabbri M, Grimaldi A, Anselmo A, Inforzato A, Collotta A et al. 2013 Loss of function of Ribonuclease T2, an ancient and phylogenetically conserved RNase, plays a crucial role in ovarian tumorigenesis. PNAS $\mathbf{1 1 0}$ 8140-8145. (https://doi.org/10.1073/pnas.1222079110)

Agarwal S, Saini S, Parashar D, Verma A, Jagadish N, Batra A, Suri S, Bhatnagar A, Gupta A, Ansari AS et al. 2013 Expression and humoral response of A-kinase anchor protein 4 in cervical cancer. International Journal of Gynecological Cancer 23 650-658. (https://doi.org/10.1097/ IGC.0b013e31828a0698)

Buck J, Sinclair ML, Schapal L, Cann MJ \& Levin LR 1999 Cytosolic adenylyl cyclase defines a unique signaling molecule in mammals. PNAS 96 79-84. (https://doi.org/10.1073/pnas.96.1.79)

Campomenosi P, Salis S, Lindqvist C, Mariani D, Nordström T, Acquati F \& Taramelli R 2006 Characterization of RNASET2, the first human member of the Rh/T2/S family of glycoproteins. Archives of Biochemistry and Biophysics 449 17-26. (https://doi.org/10.1016/j.abb.2006.02.022)

Chen Y, Cann MJ, Litvin TN, lourgenko V, Sinclair ML, Levin LR \& Buck J 2000 Soluble adenylyl cyclase as an evolutionarily conserved bicarbonate sensor. Science 289 625-628. (https://doi.org/10.1126/ science.289.5479.625)

Chiriva-Internati M, Ferrari R, Yu Y, Hamrick C, Gagliano N, Grizzi F, Frezza E, Jenkins MR, Hardwick F, D'Cunha N et al. 2008 AKAP4: a novel cancer testis antigen for multiple myeloma. British Journal of Haematology $\mathbf{1 4 0} 465-468 . \quad$ (https://doi.org/10.1111/j.13652141.2007.06940.x)

Chiriva-Internati M, Yu Y, Mirandola L, D'Cunha N, Hardwicke F, Cannon MJ, Cobos E \& Kast WM 2012 Identification of AKAP-4 as a new cancer/testis antigen for detection and immunotherapy of prostate cancer. Prostate 72 12-23. (https://doi.org/10.1002/pros.21400) de Leeuw M, González A, Lanir A, Roiz L, Smirnoff P, Schwartz B, Shoseyov O \& Almog O 2012 The 1.8 A crystal structure of ACTIBIND suggests a mode of action for $\mathrm{T} 2$ ribonucleases as antitumorigenic agents. Journal of Medicinal Chemistry 55 1013-1020. (https://doi.org/10.1021/ jm1015507)

Eddy EM, Toshimori K \& O'Brien DA 2003 Fibrous sheath of mammalian spermatozoa. Microscopy Research and Technique 61 103-115. (https:// doi.org/10.1002/jemt.10320)

Esposito G, Jaiswal BS, Xie F, Krajnc-Franken MA, Robben TJ, Strik AM, Kuil C, Philipsen RL, van Duin M, Conti M et al. 2004 Mice deficient for soluble adenylyl cyclase are infertile because of a severe spermmotility defect. PNAS 101 2993-2998. (https://doi.org/10.1073/ pnas.0400050101)

Gallardo Bolanos JM, Balao da Silva CM, Martín Muñoz P, Morillo Rodríguez A, Plaza Dávila M, Rodríguez-Martínez H, Aparicio IM, Tapia JA, Ortega Ferrusola C \& Peña FJ 2014 Phosphorylated AKT preserves stallion sperm viability and motility by inhibiting caspases 3 and 7. Reproduction 148 221-235. (https://doi.org/10.1530/REP-13-0191)

Gumireddy K, Li A, Chang DH, Liu Q, Kossenkov AV, Yan J, Korst RJ, Nam BT, Xu H, Zhang L et al. 2015 AKAP4 is a circulating biomarker for non-small cell lung cancer. Oncotarget 6 17637-17647. (https;//doi. org/10.18632/oncotarget.3946)

Hess KC, Jones BH, Marquez B, Chen Y, Ord TS, Kamenetsky M, Miyamoto C, Zippin JH, Kopf GS, Suarez SS et al. 2005 The 'soluble' adenylyl cyclase in sperm mediates multiple signaling events required for fertilization. Developmental Cell 9 249-259. (https://doi.org/10.1016/j. devcel.2005.06.007)

Ignotz GG \& Suarez SS 2005 Calcium/calmodulin and calmodulin kinase II stimulate hyperactivation in demembranated bovine sperm. Biology of Reproduction 73 519-526. (https://doi.org/10.1095/ biolreprod.105.040733)

Jagadish N, Parashar D, Gupta N, Agarwal S, Sharma A, Fatima R, Suri V, Kumar R, Gupta A, Lohiya NK et al. 2016 A novel cancer testis antigen target A-kinase anchor protein (AKAP4) for the early diagnosis and immunotherapy of colon cancer. Oncoimmunology 5 e1078965. (https://doi.org/10.1080/2162402X.2015.1078965)

Jaiswal BS \& Conti M 2003 Calcium regulation of the soluble adenylyl cyclase expressed in mammalian spermatozoa. PNAS $\mathbf{1 0 0}$ 10676-10681. (https://doi.org/10.1073/pnas.1831008100)

Li S, Qin X, Li Y, Guo A, Ma L, Jiao F \& Chai S 2016 AKAP4 mediated tumor malignancy in esophageal cancer. American Journal of Translational Research 8 597-605.

Lin H \& Morin PJ 2001 A novel homozygous deletion at chromosomal band 6 q27 in an ovarian cancer cell line delineates the position of a putative tumor suppressor gene. Cancer Letters 173 63-70. (https://doi. org/10.1016/S0304-3835(01)00677-2)

Litvin TN, Kamenetsky M, Zarifyan A, Buck J \& Levin LR 2003 Kinetic properties of 'soluble' adenylyl cyclase. Synergism between calcium and bicarbonate. Journal of Biological Chemistry 278 15922-15926. (https:// doi.org/10.1074/jbc.M212475200)

Liu Y, Chen G, Lu L, Sun H, Guo Q, Xue K, Fan Y \& Ding Z 2013 RNASET2 in human spermatozoa and seminal plasma: a novel relevant indicator for asthenozoospermia. Andrology 1 75-84. (https://doi.org/10.1111/ j.2047-2927.2012.00022.x)

Liu Y, Guo Y, Song N, Fan Y, Li K, Teng X, Guo Q \& Ding Z 2015 Proteomic pattern changes associated with obesity-induced asthenozoospermia. Andrology 3 247-259. (https://doi.org/10.1111/andr.289)

Luconi M, Cantini G, Baldi E \& Forti G 2011 Role of a-kinase anchoring proteins (AKAPs) in reproduction. Frontiers in Bioscience 16 1315-1330. (https://doi.org/10.2741/3791)

Luhtala N \& Parker R 2010 T2 Family ribonucleases: ancient enzymes with diverse roles. Trends in Biochemical Sciences 35 253-259. (https://doi. org/10.1016/j.tibs.2010.02.002)

Marin-Briggiler $\mathrm{Cl}$, Jha KN, Chertihin O, Buffone MG, Herr JC, Vazquez-Levin MH \& Visconti PE 2005 Evidence of the presence of calcium / calmodulin-dependent protein kinase IV in human sperm and its involvement in motility regulation. Journal of Cell Science $\mathbf{1 1 8}$ 2013-2022. (https://doi.org/10.1242/jcs.02326)

Miki K, Willis WD, Brown PR, Goulding EH, Fulcher KD \& Eddy EM 2002 Targeted disruption of the Akap4 gene causes defects in sperm flagellum and motility. Developmental Biology 248 331-342. (https:// doi.org/10.1006/dbio.2002.0728) 
Monti L, Rodolfo M, Lo Russo G, Noonan D, Acquati F \& Taramelli R 2008 RNASET2 as a tumor antagonizing gene in a melanoma cancer model. Oncology Research 17 69-74. (https://doi. org/10.3727/096504008784523658)

Nesiel-Nuttman L, Schwartz B \& Shoseyov O 2014 Human recombinant truncated RNASET2, devoid of RNase activity; a potential cancer therapeutic agent. Oncotarget 5 11464-11478. (https;//doi. org/10.18632/oncotarget.2562)

Nesiel-Nuttman L, Doron S, Schwartz B \& Shoseyov O 2015 Human RNASET2 derivatives as potential anti-angiogenic agents: actin binding sequence identification and characterization. Oncoscience 2 31-43. (https://doi.org/10.18632/oncoscience.100)

Overstreet JW, Yanagimachi R, Katz DF, Hayashi K \& Hanson FW 1980 Penetration of human spermatozoa into the human zona pellucida and the zona-free hamster egg: a study of fertile donors and infertile patients. Fertility and Sterility 33 534-542. (https://doi.org/10.1016/S00150282(16)44720-5)

Ragel BT, Couldwell WT, Gillespie DL \& Jensen RL 2007 Identification of hypoxia-induced genes in a malignant glioma cell line (U-251) by cDNA microarray analysis. Neurosurgical Review 30 181-187. (https:// doi.org/10.1007/s10143-007-0070-z)

Roiz L, Ozeri U, Goren R \& Shoseyov O 2000 Characterization of Aspergillus niger B-1 RNase and its inhibitory effect on pollen germination and pollen tube growth in selected tree fruit. Journal of the American Society for Horticultural Science 125 9-14. (https://doi. org/10.18632/oncoscience.295)

Roiz L, Smirnoff P, Bar-Eli M, Schwartz B \& Shoseyov O 2006 ACTIBIND, an actin-binding fungal T2-RNase with antiangiogenic and anticarcinogenic characteristics. Cancer 106 2295-2308. (https://doi. org/10.1002/cncr.21878)

Roiz L, Smirnoff P, Lewin I, Shoseyov O \& Schwartz B 2016 Human recombinant RNASET2: A potential anti-cancer drug. Oncoscience 3 71-84.

Saini S, Jagadish N, Gupta A, Bhatnagar A \& Suri A 2013 A novel cancer testis antigen, A-kinase anchor protein 4 (AKAP4) is a potential biomarker for breast cancer. PLOS ONE 8 e57095. (https://doi.org/10.1371/journal. pone.0057095)

Schwartz B, Shoseyov O, Melnikova VO, McCarty M, Leslie M, Roiz L, Smirnoff P, Hu GF, Lev D \& Bar-Eli M 2007 ACTIBIND, a T2 RNase, competes with angiogenin and inhibits human melanoma growth, angiogenesis, and metastasis. Cancer Research 67 5258-5266. (https:// doi.org/10.1158/0008-5472.CAN-07-0129)

Smirnoff P, Roiz L, Angelkovitch B, Schwartz B \& Shoseyov O 2006 A recombinant human RNASET2 glycoprotein with antitumorigenic and antiangiogenic characteristics: expression, purification, and characterization. Cancer 107 2760-2769. (https://doi.org/10.1002/ cncr.22327)

Suarez SS \& Osman RA 1987 Initiation of hyperactivated flagellar bending in mouse sperm within the female reproductive tract. Biology of Reproduction 36 1191-1198. (https://doi.org/10.1095/ biolreprod36.5.1191)

Tash JS \& Means AR 1982 Regulation of protein phosphorylation and motility of sperm by cyclic adenosine monophosphate and calcium. Biology of Reproduction 26 745-763. (https://doi.org/10.1095/ biolreprod26.4.745)

Tash JS \& Means AR 1983 Cyclic adenosine 3',5' monophosphate, calcium and protein phosphorylation in flagellar motility. Biology of Reproduction 28 75-104. (https://doi.org/10.1095/biolreprod28.1.75)

Turner RM 2006 Moving to the beat: a review of mammalian sperm motility regulation. Reproduction Fertility and Development 18 25-38. (https://doi.org/10.1071/RD05120)

WHO 2010 Laboratory Manual for the Examination and Processing of Human Semen. In World Health Organization, edn 5, pp 10-26. Eds TG Cooper. Geneva: World Health Organization.

Wu JY, Ribar TJ, Cummings DE, Burton KA, McKnight GS \& Means AR 2000 Spermiogenesis and exchange of basic nuclear proteins are impaired in male germ cells lacking Camk4. Nature Genetics 25 448-452. (https://doi.org/10.1038/78153)

Received 11 December 2017

First decision 22 January 2018

Revised manuscript received 26 January 2018

Accepted 13 February 2018 